\title{
Research Article \\ Fuzzy Stability of Additive Functional Inequalities with the Fixed Point Alternative
}

\author{
Choonkil Park \\ Department of Mathematics, Research Institute for Natural Sciences, Hanyang University, Seoul 133-791, \\ South Korea \\ Correspondence should be addressed to Choonkil Park, baak@hanyang.ac.kr \\ Received 12 October 2009; Revised 16 November 2009; Accepted 30 November 2009 \\ Academic Editor: Nikolaos Papageorgiou \\ Copyright ( $) 2009$ Choonkil Park. This is an open access article distributed under the Creative \\ Commons Attribution License, which permits unrestricted use, distribution, and reproduction in \\ any medium, provided the original work is properly cited. \\ Using the fixed point method, we prove the generalized Hyers-Ulam stability of the Cauchy \\ additive functional inequality and of the Cauchy-Jensen additive functional inequality in fuzzy \\ Banach spaces.
}

\section{Introduction and Preliminaries}

Katsaras [1] defined a fuzzy norm on a vector space to construct a fuzzy vector topological structure on the space. Some mathematicians have defined fuzzy norms on a vector space from various points of view [2-4]. In particular, Bag and Samanta [5], following Cheng and Mordeson [6], gave an idea of fuzzy norm in such a manner that the corresponding fuzzy metric is of Kramosil and Michálek type [7]. They established a decomposition theorem of a fuzzy norm into a family of crisp norms and investigated some properties of fuzzy normed spaces [8].

We use the definition of fuzzy normed spaces given in $[5,9,10]$ to investigate a fuzzy version of the generalized Hyers-Ulam stability for the Cauchy additive functional inequality and for the Cauchy-Jensen additive functional inequality in the fuzzy normed vector space setting.

Definition 1.1 (see $[5,9-11]$ ). Let $X$ be a real vector space. A function $N: X \times \mathbb{R} \rightarrow[0,1]$ is called a fuzzy norm on $X$ if for all $x, y \in X$ and all $s, t \in \mathbb{R}$,

$$
\begin{aligned}
& \left(N_{1}\right) N(x, t)=0 \text { for } t \leq 0 \\
& \left(N_{2}\right) x=0 \text { if and only if } N(x, t)=1 \text { for all } t>0 \\
& \left(N_{3}\right) N(c x, t)=N(x, t /|c|) \text { if } c \neq 0
\end{aligned}
$$


$\left(N_{4}\right) N(x+y, s+t) \geq \min \{N(x, s), N(y, t)\} ;$

$\left(N_{5}\right) N(x, \cdot)$ is a nondecreasing function of $\mathbb{R}$ and $\lim _{t \rightarrow \infty} N(x, t)=1$;

$\left(N_{6}\right)$ for $x \neq 0, N(x, \cdot)$ is continuous on $\mathbb{R}$.

The pair $(X, N)$ is called a fuzzy normed vector space. in $[10,11]$.

The properties of fuzzy normed vector spaces and examples of fuzzy norms are given

Definition 1.2 (see $[5,9-11])$. Let $(X, N)$ be a fuzzy normed vector space. A sequence $\left\{x_{n}\right\}$ in $X$ is said to be convergent or converge if there exists an $x \in X$ such that $\lim _{n \rightarrow \infty} N\left(x_{n}-x, t\right)=1$ for all $t>0$. In this case, $x$ is called the limit of the sequence $\left\{x_{n}\right\}$ and we denote it by $N$ $\lim _{n \rightarrow \infty} x_{n}=x$.

Definition 1.3 (see $[5,9,10])$. Let $(X, N)$ be a fuzzy normed vector space. A sequence $\left\{x_{n}\right\}$ in $X$ is called Cauchy if for each $\varepsilon>0$ and each $t>0$ there exists an $n_{0} \in \mathbb{N}$ such that for all $n \geq n_{0}$ and all $p>0$, we have $N\left(x_{n+p}-x_{n}, t\right)>1-\varepsilon$.

It is well known that every convergent sequence in a fuzzy normed vector space is Cauchy. If each Cauchy sequence is convergent, then the fuzzy norm is said to be complete and the fuzzy normed vector space is called a fuzzy Banach space.

We say that a mapping $f: X \rightarrow Y$ between fuzzy normed vector spaces $X$ and $Y$ is continuous at a point $x_{0} \in X$ if for each sequence $\left\{x_{n}\right\}$ converging to $x_{0}$ in $X$, then the sequence $\left\{f\left(x_{n}\right)\right\}$ converges to $f\left(x_{0}\right)$. If $f: X \rightarrow Y$ is continuous at each $x \in X$, then $f: X \rightarrow$ $Y$ is said to be continuous on $X$ (see [8]).

The stability problem of functional equations originated from a question of Ulam [12] concerning the stability of group homomorphisms. Hyers [13] gave a first affirmative partial answer to the question of Ulam for Banach spaces. Hyers' theorem was generalized by Aoki [14] for additive mappings and by Th. M. Rassias [15] for linear mappings by considering an unbounded Cauchy difference. The paper of Th. M. Rassias [15] has provided a lot of influence in the development of what we call generalized Hyers-Ulam stability or as HyersUlam-Rassias stability of functional equations. A generalization of the Th. M. Rassias theorem was obtained by Găvruţa [16] by replacing the unbounded Cauchy difference by a general control function in the spirit of Th. M. Rassias' approach.

The functional equation

$$
f(x+y)+f(x-y)=2 f(x)+2 f(y)
$$

is called a quadratic mapping equation. In particular, every solution of the quadratic functional equation is said to be a quadratic function. A generalized Hyers-Ulam stability problem for the quadratic functional equation was proved by Skof [17] for mappings $f: X \rightarrow Y$, where $X$ is a normed space and $Y$ is a Banach space. Cholewa [18] noticed that the theorem of Skof is still true if the relevant domain $X$ is replaced by an Abelian group. Czerwik [19] proved the generalized Hyers-Ulam stability of the quadratic functional equation.

In [20], Jun and Kim considered the following cubic functional equation:

$$
f(2 x+y)+f(2 x-y)=2 f(x+y)+2 f(x-y)+12 f(x)
$$


which is called a cubic functional equation and every solution of the cubic functional equation is said to be a cubic mapping. In [21], Lee et al. considered the following quartic functional equation:

$$
f(2 x+y)+f(2 x-y)=4 f(x+y)+4 f(x-y)+24 f(x)-6 f(y)
$$

which is called a quartic functional equation and every solution of the quartic functional equation is said to be a quartic mapping. Quartic functional equations have been investigated in $[22,23]$.

Surveys of expository results on related advances both in single variables and in multivariables have been given in [24, 25]. The stability problems of several functional equations have been extensively investigated by a number of authors and there are many interesting results concerning this problem (see [26-33]).

Gilányi [34] showed that if $f$ satisfies the functional inequality

$$
\|2 f(x)+2 f(y)-f(x-y)\| \leq\|f(x+y)\|
$$

then $f$ satisfies the Jordan-von Neumann functional equation

$$
2 f(x)+2 f(y)=f(x+y)+f(x-y) .
$$

See also [35]. Fechner [36] and Gilányi [37] proved the generalized Hyers-Ulam stability of the functional inequality (1.4). Park et al. [38] investigated the Cauchy additive functional inequality

$$
\|f(x)+f(y)+f(z)\| \leq\|f(x+y+z)\|
$$

and the Cauchy-Jensen additive functional inequality

$$
\|f(x)+f(y)+f(2 z)\| \leq\left\|2 f\left(\frac{x+y}{2}+z\right)\right\|
$$

and proved the generalized Hyers-Ulam stability of the functional inequalities (1.6) and (1.7) in Banach spaces.

Let $X$ be a set. A function $d: X \times X \rightarrow[0, \infty]$ is called a generalized metric on $X$ if $d$ satisfies

(1) $d(x, y)=0$ if and only if $x=y$;

(2) $d(x, y)=d(y, x)$ for all $x, y \in X$;

(3) $d(x, z) \leq d(x, y)+d(y, z)$ for all $x, y, z \in X$.

We recall a fundamental result in fixed point theory. 
Theorem 1.4 (see $[39,40])$. Let $(X, d)$ be a complete generalized metric space and let $J: X \rightarrow X$ be a strictly contractive mapping with Lipschitz constant $L<1$. Then for each given element $x \in X$, either

$$
d\left(J^{n} x, J^{n+1} x\right)=\infty
$$

for all nonnegative integers $n$ or there exists a positive integer $n_{0}$ such that

(1) $d\left(J^{n} x, J^{n+1} x\right)<\infty, \forall n \geq n_{0}$;

(2) the sequence $\left\{J^{n} x\right\}$ converges to a fixed point $y^{*}$ of $J$;

(3) $y^{*}$ is the unique fixed point of $J$ in the set $Y=\left\{y \in X \mid d\left(J^{n_{0}} x, y\right)<\infty\right\}$;

(4) $d\left(y, y^{*}\right) \leq(1 /(1-L)) d(y, J y)$ for all $y \in Y$.

In 1996, Isac and Th. M. Rassias [41] were the first to provide applications of stability theory of functional equations for the proof of new fixed point theorems with applications. By using fixed point methods, the stability problems of several functional equations have been extensively investigated by a number of authors (see [42-47]).

The generalized Hyers-Ulam stability of different functional equations in random normed spaces and in probabilistic normed spaces has been recently studied in [48-52].

In [53], Park et al. proved the generalized Hyers-Ulam stability of the functional inequalities (1.6) and (1.7) in fuzzy Banach spaces in the spirit of Hyers, Ulam, and Th. M. Rassias.

This paper is organized as follows. In Section 2, using the fixed point method, we prove the generalized Hyers-Ulam stability of the Cauchy additive functional inequality (1.6) in fuzzy Banach spaces. In Section 3, using fixed point method, we prove the generalized Hyers-Ulam stability of the Cauchy-Jensen additive functional inequality (1.7) in fuzzy Banach spaces.

Throughout this paper, assume that $X$ is a vector space and that $(Y, N)$ is a fuzzy Banach space.

\section{Fuzzy Stability of the Cauchy Additive Functional Inequality}

In this section, using the fixed point method, we prove the generalized Hyers-Ulam stability of the Cauchy additive functional inequality (1.6) in fuzzy Banach spaces.

Theorem 2.1. Let $\varphi: X^{3} \rightarrow[0, \infty)$ be a function such that there exists an $L<1$ with

$$
\varphi(x, y, z) \leq \frac{L}{2} \varphi(2 x, 2 y, 2 z)
$$

for all $x, y, z \in X$. Let $f: X \rightarrow Y$ be an odd mapping satisfying

$$
N(f(x)+f(y)+f(z), t) \geq \min \left\{N\left(f(x+y+z), \frac{t}{2}\right), \frac{t}{t+\varphi(x, y, z)}\right\}
$$


for all $x, y, z \in X$ and all $t>0$. Then $A(x):=N-\lim _{n \rightarrow \infty} 2^{n} f\left(x / 2^{n}\right)$ exists for each $x \in X$ and defines an additive mapping $A: X \rightarrow Y$ such that

$$
N(f(x)-A(x), t) \geq \frac{(2-2 L) t}{(2-2 L) t+L \varphi(x, x,-2 x)}
$$

for all $x \in X$ and all $t>0$.

Proof. Since $f$ is odd, $f(0)=0$. So $N(f(0), t / 2)=1$. Letting $y=x$ and replacing $z$ by $-2 x$ in (2.2), we get

$$
N(f(2 x)-2 f(x), t) \geq \frac{t}{t+\varphi(x, x,-2 x)}
$$

for all $x \in X$.

Consider the set

$$
S:=\{g: X \rightarrow Y\}
$$

and introduce the generalized metric on $S$ :

$$
d(g, h)=\inf \left\{\mu \in \mathbb{R}_{+}: N(g(x)-h(x), \mu t) \geq \frac{t}{t+\varphi(x, x,-2 x)}, \forall x \in X, \forall t>0\right\}
$$

where, as usual, inf $\phi=+\infty$. It is easy to show that $(S, d)$ is complete. (See the proof of Lemma 2.1 of [49].)

Now we consider the linear mapping $J: S \rightarrow S$ such that

$$
J g(x):=2 g\left(\frac{x}{2}\right)
$$

for all $x \in X$.

Let $g, h \in S$ be given such that $d(g, h)=\varepsilon$. Then

$$
N(g(x)-h(x), \varepsilon t) \geq \frac{t}{t+\varphi(x, x,-2 x)}
$$


for all $x \in X$ and all $t>0$. Hence

$$
\begin{aligned}
N(J g(x)-J h(x), L \varepsilon t) & =N\left(2 g\left(\frac{x}{2}\right)-2 h\left(\frac{x}{2}\right), L \varepsilon t\right) \\
& =N\left(g\left(\frac{x}{2}\right)-h\left(\frac{x}{2}\right), \frac{L}{2} \varepsilon t\right) \\
& \geq \frac{L t / 2}{L t / 2+\varphi(x / 2, x / 2,-x)} \geq \frac{L t / 2}{L t / 2+(L / 2) \varphi(x, x,-2 x)} \\
& =\frac{t}{t+\varphi(x, x,-2 x)}
\end{aligned}
$$

for all $x \in X$ and all $t>0$. So $d(g, h)=\varepsilon$ implies that $d(J g, J h) \leq L \varepsilon$. This means that

$$
d(J g, J h) \leq L d(g, h)
$$

for all $g, h \in S$.

It follows from (2.4) that

$$
N\left(f(x)-2 f\left(\frac{x}{2}\right), \frac{L}{2} t\right) \geq \frac{t}{t+\varphi(x, x,-2 x)}
$$

for all $x \in X$ and all $t>0$. So $d(f, J f) \leq L / 2$.

By Theorem 1.4, there exists a mapping $A: X \rightarrow Y$ satisfying the following.

(1) $A$ is a fixed point of $J$, that is,

$$
A\left(\frac{x}{2}\right)=\frac{1}{2} A(x)
$$

for all $x \in X$. Since $f: X \rightarrow Y$ is odd, $A: X \rightarrow Y$ is an odd mapping. The mapping $A$ is a unique fixed point of $J$ in the set

$$
M=\{g \in S: d(f, g)<\infty\}
$$

This implies that $A$ is a unique mapping satisfying (2.12) such that there exists a $\mu \in(0, \infty)$ satisfying

$$
N(f(x)-A(x), \mu t) \geq \frac{t}{t+\varphi(x, x,-2 x)}
$$

for all $x \in X$. 
Journal of Inequalities and Applications

(2) $d\left(J^{n} f, A\right) \rightarrow 0$ as $n \rightarrow \infty$. This implies the equality

$$
N-\lim _{n \rightarrow \infty} 2^{n} f\left(\frac{x}{2^{n}}\right)=A(x)
$$

for all $x \in X$.

(3) $d(f, A) \leq(1 /(1-L)) d(f, J f)$, which implies the inequality

$$
d(f, A) \leq \frac{L}{2-2 L}
$$

This implies that the inequality (2.3) holds.

By (2.2),

$$
\begin{aligned}
& N\left(2^{n}\left(f\left(\frac{x}{2^{n}}\right)+f\left(\frac{y}{2^{n}}\right)+f\left(\frac{z}{2^{n}}\right)\right), 2^{n} t\right) \\
& \quad \geq \min \left\{N\left(2^{n} f\left(\frac{x+y+z}{2^{n}}\right), 2^{n-1} t\right), \frac{t}{t+\varphi\left(x / 2^{n}, y / 2^{n}, z / 2^{n}\right)}\right\}
\end{aligned}
$$

for all $x, y, z \in X$, all $t>0$ and all $n \in \mathbb{N}$. So

$$
\begin{aligned}
& N\left(2^{n}\left(f\left(\frac{x}{2^{n}}\right)+f\left(\frac{y}{2^{n}}\right)+f\left(\frac{z}{2^{n}}\right)\right), t\right) \\
& \quad \geq \min \left\{N\left(2^{n} f\left(\frac{x+y+z}{2^{n}}\right), \frac{t}{2}\right), \frac{t / 2^{n}}{t / 2^{n}+\left(L^{n} / 2^{n}\right) \varphi(x, y, z)}\right\}
\end{aligned}
$$

for all $x, y, z \in X$, all $t>0$ and all $n \in \mathbb{N}$. Since $\lim _{n \rightarrow \infty}\left(\left(t / 2^{n}\right) /\left(\left(t / 2^{n}\right)+\left(L^{n} / 2^{n}\right) \varphi(x, y, z)\right)\right)=1$ for all $x, y, z \in X$ and all $t>0$,

$$
N(A(x)+A(y)+A(z), t) \geq N\left(A(x+y+z), \frac{t}{2}\right)
$$

for all $x, y, z \in X$ and all $t>0$. By [53, Lemma 2.1], the mapping $A: X \rightarrow Y$ is Cauchy additive, as desired.

Corollary 2.2. Let $\theta \geq 0$ and let $p$ be a real number with $p>1$. Let $X$ be a normed vector space with norm $\|\cdot\|$. Let $f: X \rightarrow Y$ be an odd mapping satisfying

$$
N(f(x)+f(y)+f(z), t) \geq \min \left\{N\left(f(x+y+z), \frac{t}{2}\right), \frac{t}{t+\theta\left(\|x\|^{p}+\|y\|^{p}+\|z\|^{p}\right)}\right\}
$$


for all $x, y, z \in X$ and all $t>0$. Then $A(x):=N-\lim _{n \rightarrow \infty} 2^{n} f\left(x / 2^{n}\right)$ exists for each $x \in X$ and defines an additive mapping $A: X \rightarrow Y$ such that

$$
N(f(x)-A(x), t) \geq \frac{\left(2^{p}-2\right) t}{\left(2^{p}-2\right) t+\left(2+2^{p}\right) \theta\|x\|^{p}}
$$

for all $x \in X$ and all $t>0$.

Proof. The proof follows from Theorem 2.1 by taking

$$
\varphi(x, y):=\theta\left(\|x\|^{p}+\|y\|^{p}+\|z\|^{p}\right)
$$

for all $x, y, z \in X$. Then we can choose $L=2^{1-p}$ and we get the desired result.

Theorem 2.3. Let $\varphi: X^{3} \rightarrow[0, \infty)$ be a function such that there exists an $L<1$ with

$$
\varphi(x, y, z) \leq 2 L \varphi\left(\frac{x}{2}, \frac{y}{2}, \frac{z}{2}\right)
$$

for all $x, y, z \in X$. Let $f: X \rightarrow Y$ be an odd mapping satisfying (2.2). Then $A(x):=N$ $\lim _{n \rightarrow \infty}\left(1 / 2^{n}\right) f\left(2^{n} x\right)$ exists for each $x \in X$ and defines an additive mapping $A: X \rightarrow Y$ such that

$$
N(f(x)-A(x), t) \geq \frac{(2-2 L) t}{(2-2 L) t+\varphi(x, x,-2 x)}
$$

for all $x \in X$ and all $t>0$.

Proof. Let $(S, d)$ be the generalized metric space defined in the proof of Theorem 2.1.

Consider the linear mapping $J: S \rightarrow S$ such that

$$
J g(x):=\frac{1}{2} g(2 x)
$$

for all $x \in X$.

Let $g, h \in S$ be given such that $d(g, h)=\varepsilon$. Then

$$
N(g(x)-h(x), \varepsilon t) \geq \frac{t}{t+\varphi(x, x,-2 x)}
$$


for all $x \in X$ and all $t>0$. Hence

$$
\begin{aligned}
N(J g(x)-J h(x), L \varepsilon t) & =N\left(\frac{1}{2} g(2 x)-\frac{1}{2} h(2 x), L \varepsilon t\right) \\
& =N(g(2 x)-h(2 x), 2 L \varepsilon t) \\
& \geq \frac{2 L t}{2 L t+\varphi(2 x, 2 x,-4 x)} \geq \frac{2 L t}{2 L t+2 L \varphi(x, x,-2 x)} \\
& =\frac{t}{t+\varphi(x, x,-2 x)}
\end{aligned}
$$

for all $x \in X$ and all $t>0$. So $d(g, h)=\varepsilon$ implies that $d(J g, J h) \leq L \varepsilon$. This means that

$$
d(J g, J h) \leq L d(g, h)
$$

for all $g, h \in S$.

It follows from (2.4) that

$$
N\left(f(x)-\frac{1}{2} f(2 x), \frac{1}{2} t\right) \geq \frac{t}{t+\varphi(x, x,-2 x)}
$$

for all $x \in X$ and all $t>0$. So $d(f, J f) \leq 1 / 2$.

By Theorem 1.4, there exists a mapping $A: X \rightarrow Y$ satisfying the following.

(1) $A$ is a fixed point of $J$, that is,

$$
A(2 x)=2 A(x)
$$

for all $x \in X$. Since $f: X \rightarrow Y$ is odd, $A: X \rightarrow Y$ is an odd mapping. The mapping $A$ is a unique fixed point of $J$ in the set

$$
M=\{g \in S: d(f, g)<\infty\}
$$

This implies that $A$ is a unique mapping satisfying (2.30) such that there exists a $\mu \in(0, \infty)$ satisfying

$$
N(f(x)-A(x), \mu t) \geq \frac{t}{t+\varphi(x, x,-2 x)}
$$

for all $x \in X$.

(2) $d\left(J^{n} f, A\right) \rightarrow 0$ as $n \rightarrow \infty$. This implies the equality

$$
N-\lim _{n \rightarrow \infty} \frac{1}{2^{n}} f\left(2^{n} x\right)=A(x)
$$

for all $x \in X$. 
(3) $d(f, A) \leq(1 /(1-L)) d(f, J f)$, which implies the inequality

$$
d(f, A) \leq \frac{1}{2-2 L}
$$

This implies that the inequality (2.24) holds.

The rest of the proof is similar to the proof of Theorem 2.1.

Corollary 2.4. Let $\theta \geq 0$ and let $p$ be a real number with $0<p<1$. Let $X$ be a normed vector space with norm $\|\cdot\|$. Let $f: X \rightarrow Y$ be an odd mapping satisfying (2.20). Then $A(x):=N$ $\lim _{n \rightarrow \infty}\left(1 / 2^{n}\right) f\left(2^{n} x\right)$ exists for each $x \in X$ and defines an additive mapping $A: X \rightarrow Y$ such that

$$
N(f(x)-A(x), t) \geq \frac{\left(2-2^{p}\right) t}{\left(2-2^{p}\right) t+\left(2+2^{p}\right) \theta\|x\|^{p}}
$$

for all $x \in X$ and all $t>0$.

Proof. The proof follows from Theorem 2.3 by taking

$$
\varphi(x, y):=\theta\left(\|x\|^{p}+\|y\|^{p}+\|z\|^{p}\right)
$$

for all $x, y, z \in X$. Then we can choose $L=2^{p-1}$ and we get the desired result.

\section{Fuzzy Stability of the Cauchy-Jensen Additive Functional Inequality}

In this section, using the fixed point method, we prove the generalized Hyers-Ulam stability of the Cauchy-Jensen additive functional inequality (1.7) in fuzzy Banach spaces.

Theorem 3.1. Let $\varphi: X^{3} \rightarrow[0, \infty)$ be a function such that there exists an $L<1$ with

$$
\varphi(x, y, z) \leq \frac{L}{2} \varphi(2 x, 2 y, 2 z)
$$

for all $x, y, z \in X$. Let $f: X \rightarrow Y$ be an odd mapping satisfying

$$
N(f(x)+f(y)+f(2 z), t) \geq \min \left\{N\left(2 f\left(\frac{x+y}{2}+z\right), \frac{2 t}{3}\right), \frac{t}{t+\varphi(x, y, z)}\right\}
$$

for all $x, y, z \in X$ and all $t>0$. Then $A(x):=N-\lim _{n \rightarrow \infty} 2^{n} f\left(x / 2^{n}\right)$ exists for each $x \in X$ and defines an additive mapping $A: X \rightarrow Y$ such that

$$
N(f(x)-A(x), t) \geq \frac{(2-2 L) t}{(2-2 L) t+L \varphi(x, x,-x)}
$$

for all $x \in X$ and all $t>0$. 
Journal of Inequalities and Applications

Proof. Letting $y=x=-z$ in (3.2), we get

$$
N(f(2 x)-2 f(x), t) \geq \frac{t}{t+\varphi(x, x,-x)}
$$

for all $x \in X$.

Consider the set

$$
S:=\{g: X \rightarrow Y\}
$$

and introduce the generalized metric on $S$ :

$$
d(g, h)=\inf \left\{\mu \in \mathbb{R}_{+}: N(g(x)-h(x), \mu t) \geq \frac{t}{t+\varphi(x, x,-x)}, \forall x \in X, \forall t>0\right\},
$$

where, as usual, $\inf \phi=+\infty$. It is easy to show that $(S, d)$ is complete. (See the proof of Lemma 2.1 of [49].)

Now we consider the linear mapping $J: S \rightarrow S$ such that

$$
J g(x):=2 g\left(\frac{x}{2}\right)
$$

for all $x \in X$.

Let $g, h \in S$ be given such that $d(g, h)=\varepsilon$. Then

$$
N(g(x)-h(x), \varepsilon t) \geq \frac{t}{t+\varphi(x, x,-x)}
$$

for all $x \in X$ and all $t>0$. Hence

$$
\begin{aligned}
N(J g(x)-J h(x), L \varepsilon t) & =N\left(2 g\left(\frac{x}{2}\right)-2 h\left(\frac{x}{2}\right), L \varepsilon t\right) \\
& =N\left(g\left(\frac{x}{2}\right)-h\left(\frac{x}{2}\right), \frac{L}{2} \varepsilon t\right) \\
& \geq \frac{L t / 2}{(L t / 2)+\varphi(x / 2, x / 2,-x / 2)} \geq \frac{L t / 2}{L t / 2+(L / 2) \varphi(x, x,-x)} \\
& =\frac{t}{t+\varphi(x, x,-x)}
\end{aligned}
$$

for all $x \in X$ and all $t>0$. So $d(g, h)=\varepsilon$ implies that $d(J g, J h) \leq L \varepsilon$. This means that

$$
d(J g, J h) \leq L d(g, h)
$$

for all $g, h \in S$. 
It follows from (3.4) that

$$
N\left(f(x)-2 f\left(\frac{x}{2}\right), \frac{L}{2} t\right) \geq \frac{t}{t+\varphi(x, x,-x)}
$$

for all $x \in X$ and all $t>0$. So $d(f, J f) \leq L / 2$.

By Theorem 1.4, there exists a mapping $A: X \rightarrow Y$ satisfying the following.

(1) $A$ is a fixed point of $J$, that is,

$$
A\left(\frac{x}{2}\right)=\frac{1}{2} A(x)
$$

for all $x \in X$. Since $f: X \rightarrow Y$ is odd, $A: X \rightarrow Y$ is an odd mapping. The mapping $A$ is a unique fixed point of $J$ in the set

$$
M=\{g \in S: d(f, g)<\infty\} .
$$

This implies that $A$ is a unique mapping satisfying (3.12) such that there exists a $\mu \in(0, \infty)$ satisfying

$$
N(f(x)-A(x), \mu t) \geq \frac{t}{t+\varphi(x, x,-x)}
$$

for all $x \in X$.

(2) $d\left(J^{n} f, A\right) \rightarrow 0$ as $n \rightarrow \infty$. This implies the equality

$$
N-\lim _{n \rightarrow \infty} 2^{n} f\left(\frac{x}{2^{n}}\right)=A(x)
$$

for all $x \in X$.

(3) $d(f, A) \leq(1 /(1-L)) d(f, J f)$, which implies the inequality

$$
d(f, A) \leq \frac{L}{2-2 L}
$$

This implies that the inequality (3.3) holds.

The rest of proof is similar to the proof of Theorem 2.1.

Corollary 3.2. Let $\theta \geq 0$ and let $p$ be a real number with $p>1$. Let $X$ be a normed vector space with norm $\|\cdot\|$. Let $f: X \rightarrow Y$ be an odd mapping satisfying

$$
N(f(x)+f(y)+f(2 z), t) \geq \min \left\{N\left(f\left(\frac{x+y}{2}+z\right), \frac{2 t}{3}\right), \frac{t}{t+\theta\left(\|x\|^{p}+\|y\|^{p}+\|z\|^{p}\right)}\right\}
$$


for all $x, y, z \in X$ and all $t>0$. Then $A(x):=N-\lim _{n \rightarrow \infty} 2^{n} f\left(x / 2^{n}\right)$ exists for each $x \in X$ and defines an additive mapping $A: X \rightarrow Y$ such that

$$
N(f(x)-A(x), t) \geq \frac{\left(2^{p}-2\right) t}{\left(2^{p}-2\right) t+3 \theta\|x\|^{p}}
$$

for all $x \in X$ and all $t>0$.

Proof. The proof follows from Theorem 3.1 by taking

$$
\varphi(x, y):=\theta\left(\|x\|^{p}+\|y\|^{p}+\|z\|^{p}\right)
$$

for all $x, y, z \in X$. Then we can choose $L=2^{1-p}$ and we get the desired result.

Theorem 3.3. Let $\varphi: X^{3} \rightarrow[0, \infty)$ be a function such that there exists an $L<1$ with

$$
\varphi(x, y, z) \leq 2 L \varphi\left(\frac{x}{2}, \frac{y}{2}, \frac{z}{2}\right)
$$

for all $x, y, z \in X$. Let $f: X \rightarrow Y$ be an odd mapping satisfying (3.2). Then $A(x):=N$ $\lim _{n \rightarrow \infty}\left(1 / 2^{n}\right) f\left(2^{n} x\right)$ exists for each $x \in X$ and defines an additive mapping $A: X \rightarrow Y$ such that

$$
N(f(x)-A(x), t) \geq \frac{(2-2 L) t}{(2-2 L) t+\varphi(x, x,-x)}
$$

for all $x \in X$ and all $t>0$.

Proof. Let $(S, d)$ be the generalized metric space defined in the proof of Theorem 3.1.

Consider the linear mapping $J: S \rightarrow S$ such that

$$
J g(x):=\frac{1}{2} g(2 x)
$$

for all $x \in X$.

Let $g, h \in S$ be given such that $d(g, h)=\varepsilon$. Then

$$
N(g(x)-h(x), \varepsilon t) \geq \frac{t}{t+\varphi(x, x,-x)}
$$


for all $x \in X$ and all $t>0$. Hence

$$
\begin{aligned}
N(J g(x)-J h(x), L \varepsilon t) & =N\left(\frac{1}{2} g(2 x)-\frac{1}{2} h(2 x), L \varepsilon t\right) \\
& =N(g(2 x)-h(2 x), 2 L \varepsilon t) \\
& \geq \frac{2 L t}{2 L t+\varphi(2 x, 2 x,-2 x)} \geq \frac{2 L t}{2 L t+2 L \varphi(x, x,-x)} \\
& =\frac{t}{t+\varphi(x, x,-x)}
\end{aligned}
$$

for all $x \in X$ and all $t>0$. So $d(g, h)=\varepsilon$ implies that $d(J g, J h) \leq L \varepsilon$. This means that

$$
d(J g, J h) \leq L d(g, h)
$$

for all $g, h \in S$.

It follows from (3.4) that

$$
N\left(f(x)-\frac{1}{2} f(2 x), \frac{1}{2} t\right) \geq \frac{t}{t+\varphi(x, x,-x)}
$$

for all $x \in X$ and all $t>0$. So $d(f, J f) \leq 1 / 2$.

By Theorem 1.4, there exists a mapping $A: X \rightarrow Y$ satisfying the following.

(1) $A$ is a fixed point of $J$, that is,

$$
A(2 x)=2 A(x)
$$

for all $x \in X$. Since $f: X \rightarrow Y$ is odd, $A: X \rightarrow Y$ is an odd mapping. The mapping $A$ is a unique fixed point of $J$ in the set

$$
M=\{g \in S: d(f, g)<\infty\} .
$$

This implies that $A$ is a unique mapping satisfying (3.27) such that there exists a $\mu \in(0, \infty)$ satisfying

$$
N(f(x)-A(x), \mu t) \geq \frac{t}{t+\varphi(x, x,-x)}
$$

for all $x \in X$.

(2) $d\left(J^{n} f, A\right) \rightarrow 0$ as $n \rightarrow \infty$. This implies the equality

$$
N-\lim _{n \rightarrow \infty} \frac{1}{2^{n}} f\left(2^{n} x\right)=A(x)
$$

for all $x \in X$. 
(3) $d(f, A) \leq(1 /(1-L)) d(f, J f)$, which implies the inequality

$$
d(f, A) \leq \frac{1}{2-2 L}
$$

This implies that the inequality (3.21) holds.

The rest of the proof is similar to the proof of Theorem 2.1.

Corollary 3.4. Let $\theta \geq 0$ and let $p$ be a real number with $0<p<1$. Let $X$ be a normed vector space with norm $\|\cdot\|$. Let $f: X \rightarrow Y$ be an odd mapping satisfying (3.17). Then $A(x):=N$ $\lim _{n \rightarrow \infty}\left(1 / 2^{n}\right) f\left(2^{n} x\right)$ exists for each $x \in X$ and defines an additive mapping $A: X \rightarrow Y$ such that

$$
N(f(x)-A(x), t) \geq \frac{\left(2-2^{p}\right) t}{\left(2-2^{p}\right) t+3 \theta\|x\|^{p}}
$$

for all $x \in X$ and all $t>0$.

Proof. The proof follows from Theorem 3.3 by taking

$$
\varphi(x, y):=\theta\left(\|x\|^{p}+\|y\|^{p}+\|z\|^{p}\right)
$$

for all $x, y, z \in X$. Then we can choose $L=2^{p-1}$ and we get the desired result.

\section{Acknowledgment}

This work was supported by the Hanyang University in 2009.

\section{References}

[1] A. K. Katsaras, "Fuzzy topological vector spaces. II," Fuzzy Sets and Systems, vol. 12, no. 2, pp. 143-154, 1984.

[2] C. Felbin, "Finite-dimensional fuzzy normed linear space," Fuzzy Sets and Systems, vol. 48, no. 2, pp. 239-248, 1992.

[3] S. V. Krishna and K. K. M. Sarma, "Separation of fuzzy normed linear spaces," Fuzzy Sets and Systems, vol. 63, no. 2, pp. 207-217, 1994.

[4] J. Xiao and X. Zhu, "Fuzzy normed space of operators and its completeness," Fuzzy Sets and Systems, vol. 133, no. 3, pp. 389-399, 2003.

[5] T. Bag and S. K. Samanta, "Finite dimensional fuzzy normed linear spaces," Journal of Fuzzy Mathematics, vol. 11, no. 3, pp. 687-705, 2003.

[6] S. C. Cheng and J. N. Mordeson, "Fuzzy linear operators and fuzzy normed linear spaces," Bulletin of the Calcutta Mathematical Society, vol. 86, no. 5, pp. 429-436, 1994.

[7] I. Kramosil and J. Michálek, "Fuzzy metrics and statistical metric spaces," Kybernetika, vol. 11, no. 5, pp. 336-344, 1975.

[8] T. Bag and S. K. Samanta, "Fuzzy bounded linear operators," Fuzzy Sets and Systems, vol. 151, no. 3, pp. 513-547, 2005.

[9] A. K. Mirmostafaee, M. Mirzavaziri, and M. S. Moslehian, "Fuzzy stability of the Jensen functional equation," Fuzzy Sets and Systems, vol. 159, no. 6, pp. 730-738, 2008.

[10] A. K. Mirmostafaee and M. S. Moslehian, "Fuzzy versions of Hyers-Ulam-Rassias theorem," Fuzzy Sets and Systems, vol. 159, no. 6, pp. 720-729, 2008. 
[11] A. K. Mirmostafaee and M. S. Moslehian, "Fuzzy approximately cubic mappings," Information Sciences, vol. 178, no. 19, pp. 3791-3798, 2008.

[12] S. M. Ulam, A Collection of Mathematical Problems, Interscience Tracts in Pure and Applied Mathematics, no. 8, Interscience, New York, NY, USA, 1960.

[13] D. H. Hyers, "On the stability of the linear functional equation," Proceedings of the National Academy of Sciences of the United States of America, vol. 27, pp. 222-224, 1941.

[14] T. Aoki, "On the stability of the linear transformation in Banach spaces," Journal of the Mathematical Society of Japan, vol. 2, pp. 64-66, 1950.

[15] Th. M. Rassias, "On the stability of the linear mapping in Banach spaces," Proceedings of the American Mathematical Society, vol. 72, no. 2, pp. 297-300, 1978.

[16] P. Găvruţa, "A generalization of the Hyers-Ulam-Rassias stability of approximately additive mappings," Journal of Mathematical Analysis and Applications, vol. 184, no. 3, pp. 431-436, 1994.

[17] F. Skof, "Proprieta' locali e approssimazione di operatori," Rendiconti del Seminario Matematico e Fisico di Milano, vol. 53, pp. 113-129, 1983.

[18] P. W. Cholewa, "Remarks on the stability of functional equations," Aequationes Mathematicae, vol. 27, no. 1-2, pp. 76-86, 1984.

[19] St. Czerwik, "On the stability of the quadratic mapping in normed spaces," Abhandlungen aus dem Mathematischen Seminar der Universität Hamburg, vol. 62, pp. 59-64, 1992.

[20] K.-W. Jun and H.-M. Kim, "The generalized Hyers-Ulam-Rassias stability of a cubic functional equation," Journal of Mathematical Analysis and Applications, vol. 274, no. 2, pp. 267-278, 2002.

[21] S. H. Lee, S. M. Im, and I. S. Hwang, "Quartic functional equations," Journal of Mathematical Analysis and Applications, vol. 307, no. 2, pp. 387-394, 2005.

[22] J. K. Chung and P. K. Sahoo, "On the general solution of a quartic functional equation," Bulletin of the Korean Mathematical Society, vol. 40, no. 4, pp. 565-576, 2003.

[23] J. M. Rassias, "Solution of the Ulam stability problem for quartic mappings," Glasnik Matematički, vol. 34(54), no. 2, pp. 243-252, 1999.

[24] R. P. Agarwal, B. Xu, and W. Zhang, "Stability of functional equations in single variable," Journal of Mathematical Analysis and Applications, vol. 288, no. 2, pp. 852-869, 2003.

[25] G. L. Forti, "Hyers-Ulam stability of functional equations in several variables," Aequationes Mathematicae, vol. 50, no. 1-2, pp. 143-190, 1995.

[26] S. Czerwik, Functional Equations and Inequalities in Several Variables, World Scientific, River Edge, NJ, USA, 2002.

[27] D. H. Hyers, G. Isac, and Th. M. Rassias, Stability of Functional Equations in Several Variables, Progress in Nonlinear Differential Equations and Their Applications, 34, Birkhäuser, Boston, Mass, USA, 1998.

[28] S.-M. Jung, Hyers-Ulam-Rassias Stability of Functional Equations in Mathematical Analysis, Hadronic Press, Palm Harbor, Fla, USA, 2001.

[29] C. Park and J. Cui, "Generalized stability of $C^{*}$-ternary quadratic mappings," Abstract and Applied Analysis, vol. 2007, Article ID 23282, 6 pages, 2007.

[30] C. Park and A. Najati, "Homomorphisms and derivations in C*-algebras," Abstract and Applied Analysis, vol. 2007, Article ID 80630, 12 pages, 2007.

[31] Th. M. Rassias, "On the stability of functional equations in Banach spaces," Journal of Mathematical Analysis and Applications, vol. 251, no. 1, pp. 264-284, 2000.

[32] Th. M. Rassias, "On the stability of functional equations and a problem of Ulam," Acta Applicandae Mathematicae, vol. 62, no. 1, pp. 23-130, 2000.

[33] Th. M. Rassias and P. Šemrl, "On the behavior of mappings which do not satisfy Hyers-Ulam stability," Proceedings of the American Mathematical Society, vol. 114, no. 4, pp. 989-993, 1992.

[34] A. Gilányi, "Eine zur Parallelogrammgleichung äquivalente Ungleichung," Aequationes Mathematicae, vol. 62, no. 3, pp. 303-309, 2001.

[35] J. Rätz, "On inequalities associated with the Jordan-von Neumann functional equation," Aequationes Mathematicae, vol. 66, no. 1-2, pp. 191-200, 2003.

[36] W. Fechner, "Stability of a functional inequality associated with the Jordan-von Neumann functional equation," Aequationes Mathematicae, vol. 71, no. 1-2, pp. 149-161, 2006.

[37] A. Gilányi, "On a problem by K. Nikodem," Mathematical Inequalities E Applications, vol. 5, no. 4, pp. 707-710, 2002.

[38] C. Park, Y. S. Cho, and M.-H. Han, "Functional inequalities associated with Jordan-von Neumanntype additive functional equations," Journal of Inequalities and Applications, vol. 2007, Article ID 41820, 13 pages, 2007. 
[39] L. Cădariu and V. Radu, "Fixed points and the stability of Jensen's functional equation," Journal of Inequalities in Pure and Applied Mathematics, vol. 4, no. 1, article 4, 2003.

[40] J. B. Diaz and B. Margolis, "A fixed point theorem of the alternative, for contractions on a generalized complete metric space," Bulletin of the American Mathematical Society, vol. 74, pp. 305-309, 1968.

[41] G. Isac and Th. M. Rassias, "Stability of $\psi$-additive mappings: applications to nonlinear analysis," International Journal of Mathematics and Mathematical Sciences, vol. 19, no. 2, pp. 219-228, 1996.

[42] L. Cădariu and V. Radu, "On the stability of the Cauchy functional equation: a fixed point approach," in Iteration Theory, vol. 346 of Grazer Mathematische Berichte, pp. 43-52, Karl-Franzens-Universität Graz, Graz, Austria, 2004.

[43] L. Cădariu and V. Radu, "Fixed point methods for the generalized stability of functional equations in a single variable," Fixed Point Theory and Applications, vol. 2008, Article ID 749392, 15 pages, 2008.

[44] M. Mirzavaziri and M. S. Moslehian, "A fixed point approach to stability of a quadratic equation," Bulletin of the Brazilian Mathematical Society, vol. 37, no. 3, pp. 361-376, 2006.

[45] C. Park, "Fixed points and Hyers-Ulam-Rassias stability of Cauchy-Jensen functional equations in Banach algebras," Fixed Point Theory and Applications, vol. 2007, Article ID 50175, 15 pages, 2007.

[46] C. Park, "Generalized Hyers-Ulam stability of quadratic functional equations: a fixed point approach," Fixed Point Theory and Applications, vol. 2008, Article ID 493751, 9 pages, 2008.

[47] V. Radu, "The fixed point alternative and the stability of functional equations," Fixed Point Theory, vol. 4, no. 1, pp. 91-96, 2003.

[48] C. Alsina, "On the stability of a functional equation arising in probabilistic normed spaces," in General Inequalities, 5 (Oberwolfach, 1986), vol. 80 of Internationale Schriftenreihe zur Numerischen Mathematik, pp. 263-271, Birkhäuser, Basel, Switzerland, 1987.

[49] D. Miheț and V. Radu, "On the stability of the additive Cauchy functional equation in random normed spaces," Journal of Mathematical Analysis and Applications, vol. 343, no. 1, pp. 567-572, 2008.

[50] D. Miheţ, R. Saadati, and S. M. Vaezpour, "The stability of the quartic functional equation in random normed spaces," to appear in Acta Applicandae Mathematicae.

[51] D. Miheț, R. Saadati, and S. M. Vaezpour, "The stability of an additive functional equation in Menger probabilistic $\varphi$-normed spaces," Mathematica Slovaca. In press.

[52] R. Saadati, S. M. Vaezpour, and Y. J. Cho, "Erratum: a note to paper "On the stability of cubic mappings and quadratic mappings in random normed spaces"," Journal of Inequalities and Applications, vol. 2009, Article ID 214530, 6 pages, 2009.

[53] C. Park, J. Cho, and R. Saadati, "Fuzzy stability of functional inequalities," preprint. 